\title{
A Comparison of 2-D Moment-Based Description Techniques
}

\author{
C. Di Ruberto and A. Morgera \\ Dipartimento di Matematica e Informatica, \\ Università di Cagliari, Cagliari, Italy
}

\begin{abstract}
Moment invariants are properties of connected regions in binary images that are invariant to translation, rotation and scale. They are useful because they define a simply calculated set of region properties that can be used for shape classification and part recognition. Orthogonal moment invariants allow for accurate reconstruction of the described shape. Generic Fourier Descriptors yield spectral features and have better retrieval performance due to multi-resolution analysis in both radial and circular directions of the shape. In this paper we first compare various moment-based shape description techniques then we propose a method that, after a previous image partition into classes by morphological features, associates the appropriate technique with each class, i.e. the technique that better recognizes the images of that class. The results clearly demonstrate the effectiveness of this new method regard to described techniques.
\end{abstract}

\section{Introduction}

Moment invariants were firstly introduced to the pattern recognition community in 1962 by $\mathrm{Hu}$ [1], who employed the results of the theory of algebraic invariants and derived his seven famous invariants to rotation of 2-D objects. Since that time, numerous works have been devoted to various improvements and generalizations of Hu's invariants and also to its use in many application areas.

Dudani 2] and Belkasim [3] described their application to aircraft silhouette recognition, Wong and Hall [4], Goshtasby [5] and Flusser and Suk 6] employed moment invariants in template matching and registration of satellite images, Mukundan [78] applied them to estimate the position and the attitude of the object in 3-D space, Sluzek [9] proposed to use local moment invariants in industrial quality inspection and many authors used moment invariants for character recognition 310111213 .

Maitra [14] and Hupkens [15] made them invariant also to contrast changes, Wang [16] proposed illumination invariants particularly suitable for texture classification. Li [17] and Wong [18] presented the systems of invariants up to the orders nine and five, respectively. Most recently, Flusser 1920 has proposed a method how to derive independent sets of invariants of any orders. 
There is also a group of papers [12/21,22] that use Zernike moments to construct rotation invariants.

In this paper we present a comparison among different description techniques based on moment invariants. Geometric and algebraic moments are described in section 2. We examine orthogonal moments in section 3 . Section 4 discusses Generic Fourier Descriptor. In section 5 we test the described techniques for image indexing. In section 6 we propose a method to enhance the results obtained in previous experiments.

\section{Geometric and Algebraic Moments}

Moment invariants are useful in 2-D object recognition. Moment invariants are functions of moments that are invariant under certain transformations. Although moments are defined on a continuous image intensity function, a simple approximation is possible for a discrete binary image using summation operation. Let $f$ be a binary digital image matrix with dimension $M \times N$, and let

$$
S=\{(x, y) \mid f(x, y)=1\}
$$

represent a 2 -D shape. The moment of order $(p, q)$ of shape $S$ is given by

$$
m_{p q}(S)=\sum_{(x, y) \in S} x^{p} y^{q}
$$

The central moment of order $(p, q)$ of shape $S$ is given by

$$
\mu_{p q}(S)=\sum_{(x, y) \in S}(x-\bar{x})^{p}(y-\bar{y})^{q}
$$

where $(\bar{x}, \bar{y})$ is the center of gravity. From the central moments, the normalized central moments have been defined. From the second- and third-order normalized central moments, a set of seven invariant moments, which is invariant to translation, scale change and rotation, has been derived by $\mathrm{Hu}[23$. $\mathrm{Hu}$ has also proved the invariance properties of the seven moments for the case of continuous function. Flusser and Suk 24 derived affine moment invariants wich are invariant under general 2-D affine tranformations. In 25] Taubin and Cooper defined algebraic moment invariants by introducing the concept of covariance matrix.

\section{Orthogonal Moments}

\subsection{Zernike Moment Invariants}

There are many different types of moments that have been applied to computer vision problems, but it has been demonstrated in [26] that the orthogonal Zernike moments offer a set of moments which are highly uncorrelated with little information redundancy. The orthogonal Zernike moments, first proposed by Teague 
in [27], utilize the Zernike polynomial as basis function and are defined over the unit disc (in polar coordinates) by:

$$
Z_{m n}=\frac{m+1}{\pi} \int_{0}^{2 \pi} \int_{0}^{1} V_{m n}^{*}(r, \theta) f(r, \theta) r d r d \theta
$$

where $m$ is the order of the moment (with $m \geq 0$ ) and $n$ represents the repetition (where $|n| \leq m$, and $m+n$ is even). $V_{m n}(r, \theta)$ is the complex-valued Zernike polynomial with * indicating the complex conjugate. For a discrete square image (size $N \mathrm{x} N), Z_{m n}$ can be calculated with:

$$
Z_{m n}=\frac{m+1}{\pi} \frac{1}{(N-1)^{2}} \sum_{x=0}^{N-1} \sum_{y=0}^{N-1} V_{m n}^{*}(r, \theta) f(x, y)
$$

given that $r=\left(x^{2}+y^{2}\right)^{1 / 2} / \sqrt{2} N$ and $\theta=\tan ^{-1}(y / x)$, in order to map the image into the unit disc. Note that last equation is only orthogonal over the unit circle. The Zernike polynomial, $V_{m n}(r, \theta)$, is defined as:

$$
V_{m n}(r, \theta)=R_{m n}(r) e^{-j n \theta}=R_{m n}(r)(\cos n \theta-j \sin n \theta)
$$

where the radial polynomial, $R_{m} n(r)$, is:

$$
R_{m n}(r)=\sum_{s=0}^{m-|n| / 2} \frac{(-1)^{s}(m-s) ! r^{m-2 s}}{s !\left(\frac{m+|n|}{2}-s\right) !\left(\frac{m-|n|}{2}-s\right) !} .
$$

This polynomial is such that over the unit disc, $\left|R_{m n}(r)\right| \leq 1$ and that $R_{m n}(1)=$ 1 , for any values of $m$ and $n$. The definition of the radial polynomial also leads to $R_{m n}(r)=R_{m,-n}(r)$. The number of Zernike moments for any order, $m$, is given by $m+1$, while the number of moments up to and including order $m$ is $(m / 2+$ $1)(m+1)$, (although because of the relationship between $Z_{m n}$ and $Z_{m,-n}$ given above, only the moments with $n \geq 0$ need to be known).

\subsection{Legendre Moments}

The kernel of Legendre moments is products of Legendre polynomials defined along rectangular image coordinate axes inside a unit circle. The $(p, q)$ order Legendre moments are defined as:

$$
\lambda_{p q}=\frac{(2 p+1)(2 q+1)}{4} \int_{-\infty}^{+\infty} \int_{-\infty}^{+\infty} P_{p}(x) P_{q}(y) f(x, y) d x d y
$$

where the function $P_{p}(x)$ denote Legendre polynomial of order $p$. The Legendre moment generalizes the geometric moments in the sense that the monomial $x^{p} y^{q}$ 
is replaced by the orthogonal polynomial $P_{p}(x) P_{q}(y)$ of the same order. The discrete version of the Legendre moments can be written as:

$$
\lambda_{p q}=\frac{(2 p+1)(2 q+1)}{(M-1)(N-1)} \sum_{x=0}^{M-1} \sum_{y=0}^{N-1} p_{p}(x) p_{q}(y) f(x, y)
$$

where $(p+q)$ is the order, $p, q=0,1,2,3, \ldots, \infty$. The Legendre polynomials, $P_{p}(x)$ are a complete orthogonal basis set on the interval $[-1,1]$ :

$$
\int_{-1}^{1} p_{p}(x) p_{q}(y) d x=\frac{2}{2 p+1} \delta_{p q}
$$

The $n^{t h}-$ order Legendre polynomial are defined by:

$$
p_{q}(x)=\frac{1}{2^{q}} \sum_{p=0}^{\frac{q}{2}}(-1)^{q} \frac{(2 q-2 p) !}{p !(q-p) !(q-2 p) !} x^{q-2 p} .
$$

\section{2-D Generic Fourier Descriptors}

Fourier transform has been widely used for image processing and analysis. The advantage of analyzing image in spectral domain over analyzing shape in spatial domain is that it is easy to overcome the noise problem which is common to digital images. At first a polar transformation of an input image $f(x, y)$ is done, obtaining a polar image $f(\rho, \theta)$ by

$$
r=\sqrt{\left(x-x_{c}\right)^{2}+\left(y-y_{c}\right)^{2}}, \theta=\tan ^{-1}(y / x)
$$

where $\left(x_{c}, y_{c}\right)$ are the coordinates of centroid. Then a transformation of polar raster sampled image in Cartesian space is done. Then 2-D Fourier Transform of this polar raster sampled image $f(\rho, \theta)$ is calculated:

$$
P F_{2}(\rho, \phi)=\sum_{r} \sum_{i} f\left(r, \theta_{i}\right) \exp \left[j 2 \pi\left(\frac{r}{R} \rho+\frac{2 \pi i}{T} \phi\right)\right]
$$

where $(0 \leq l<R)$ and $\theta_{i}=i(2 \pi / T)(0 \leq i<T), 0 \leq \rho<R, 0 \leq \phi<T$. $R$ and $T$ are the resolution of radial frequency and angular frequency respectively. The normalized Fourier coefficients are the GFD.

\section{Test of Retrieval Effectiveness}

In order to test the retrieval performance of the described techniques we have constructed an image database made of 19 query shapes and 219 test shapes, composed by tools, biological and common shapes 28,29]. In figure1 the experiment images are showed : they are numbered from 1 to 19 starting from top left corner. To recognize the test images we applied the described techniques according to the following 5 steps: 


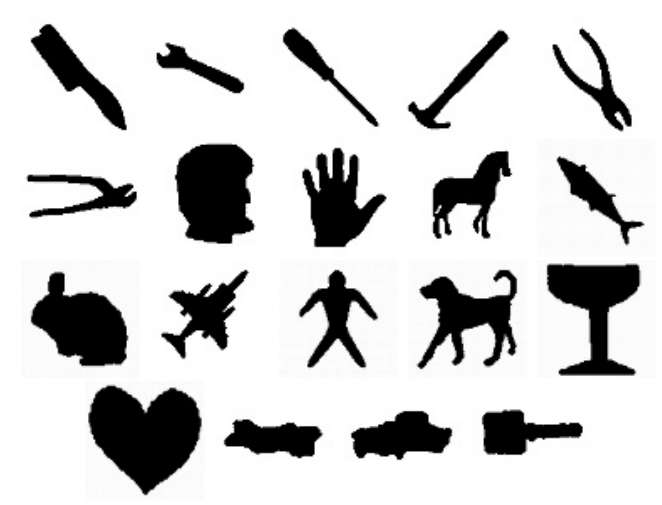

Fig. 1. Experiment shapes

1. We first create a feature vector for each query shape and each description technique. In particular, we construct such a vector by computing invariants for the image using geometric and algebraic moments based techniques. Applying orthogonal methods and GFD we obtain a coefficient matrix; in order to derive the feature vectors a linearization of this matrix for each image is done.

2. In the same way we create a feature vector for each test image.

3. By normalizing or not the feature vector, we compute the distance between the query shape feature vector and each test image feature vector; this distance has been computed both with Euclidean norm and with city-block norm. At the end of this step we have 4 measures.

4. Using a minimum distance criterion, we sort test images for each query shape.

5. If we indicate with $M$ the number of shapes of a certain class, we can calculate a hit ratio as follows:

$$
H R=\frac{\sum_{i=1}^{M} \alpha_{i}}{M}
$$

where $\alpha_{i}=1$ if the image belongs to that class, 0 otherwise.

6. As we use 4 measure distances, we obtain 4 hit ratios from which we calculate an average hit ratio for each query shape.

In table 1 you can see the indexing results. To consider higher order moments with orthogonal moments and GFD we have mediated among order $m n$ and $2 \leq m, n \leq 7$ with $n \leq m$. Computing all $m n$ orders you can obtain the maximum hit ratio for each method : Legendre 39.04\%, Zernike 57.95\%, GFD $60.13 \%$. It's important to observe that we are not too far from the global medium hit ratio. As we can notice from results, Flusser's invariants and Legendre moments have a low capability of shape recognition if we compare them to other tested methods. This fact is due to inherent limitation; in fact Legendre moments are not invariants under image rotation while Flusser's invariants worsk only with affine transformation [24]. 
Table 1. 19 query shape experiment results

\begin{tabular}{c|cccccc} 
& HU & FLU & TAU & LEG & ZER & GFD \\
\hline img1 & 0.3333 & 0.0000 & 0.1111 & 0.0972 & 0.4167 & 0.1389 \\
img2 & 0.4500 & 0.0500 & 0.6000 & 0.1250 & 0.5000 & 0.5542 \\
img3 & 0.5577 & 0.1923 & 0.3846 & 0.0961 & 0.3462 & 0.4808 \\
img4 & 0.5500 & 0.2000 & 1.0000 & 0.4667 & 0.4000 & 0.4833 \\
img5 & 0.0769 & 0.3077 & 0.3847 & 0.0994 & 0.3654 & 0.5705 \\
img6 & 0.5000 & 0.2000 & 0.2000 & 0.0750 & 0.6500 & 0.3167 \\
img7 & 0.2941 & 0.0882 & 0.1912 & 0.1814 & 0.3088 & 0.2500 \\
img8 & 0.6500 & 0.2000 & 0.6500 & 0.3250 & 1.0000 & 0.9167 \\
img9 & 0.6346 & 0.4231 & 0.3077 & 0.0449 & 0.2308 & 0.1410 \\
img10 & 0.4000 & 0.4000 & 0.1000 & 0.3792 & 0.3000 & 0.6792 \\
img11 & 0.4500 & 0.3500 & 0.5750 & 0.1167 & 0.5250 & 0.7958 \\
img12 & 0.3250 & 0.1000 & 0.4750 & 0.3458 & 0.6750 & 0.5250 \\
img13 & 0.3250 & 0.4500 & 0.2250 & 0.6250 & 0.3000 & 0.8542 \\
img14 & 0.3269 & 0.3462 & 0.1539 & 0.2532 & 0.3462 & 0.2212 \\
img15 & 0.7500 & 0.7273 & 0.5455 & 0.6212 & 0.8182 & 0.8977 \\
img16 & 0.3863 & 0.2727 & 0.7273 & 0.5909 & 0.9546 & 0.7311 \\
img17 & 0.5910 & 0.8182 & 0.9091 & 0.6137 & 0.8637 & 0.8788 \\
img18 & 0.9091 & 0.8182 & 0.7955 & 0.7652 & 0.9091 & 0.9621 \\
img19 & 0.4773 & 0.1818 & 0.7500 & 0.3977 & 0.9318 & 0.9205 \\
\hline global & 8.9872 & 6.1256 & 9.0855 & 6.2192 & 10.8413 & 11.3175 \\
perc & 47.30 & 32.24 & 47.82 & 32.73 & 57.06 & 59.57
\end{tabular}

\section{Methods Enhancement}

In order to improve the performance of the described methods, we partition images into classes so that we can associate a proper shape recognition method with each identified class. To identify the class partitioning we extract effective features of images. To do this, we consider morphological image features and other information derived from the image skeleton. We use then the following features :

- Normalized area

- Elongatedness

- Circularity

- Skeleton branch number

After feature normalization in [0:1] interval, we divide images into 6 classes with a K-means clustering algorithm. For each image we identify the method that better recognizes this shape by calculating the best medium hit ratio. Combining this choice with clustering, we decide to select a method for that class based on majority criterion. In tie case we do a minimum variance choice. For example the k-means classification assign the same class to images n.5 and n.6. The best results we obtained for image n.5 is with GFD, while for image n.6 is obtained with Zernike moments. In this case we choose Zernike moments because we obtained a lower variance. In table 2 we show numerical values of the experiment. 
Table 2. Resuming table of normalized image features, classification classes, chosen description technique

\begin{tabular}{c|cccccc} 
img N. & Normalized Area Elongatedness Circularity & \# Skel. Branch Class_\# Method \\
\hline 1 & 0.2298 & 0.4937 & 0.1150 & 0.1667 & 1 & TAU \\
2 & 0.0000 & 0.5511 & 0.2502 & 0.0833 & 1 & TAU \\
3 & 0.0174 & 1.0000 & 0.2672 & 0.0833 & 1 & TAU \\
4 & 0.0623 & 0.6720 & 0.3553 & 0.0000 & 1 & TAU \\
5 & 0.0748 & 0.1722 & 0.7309 & 0.1667 & 2 & ZER \\
6 & 0.0667 & 0.1662 & 1.0000 & 0.1667 & 2 & ZER \\
7 & 0.7484 & 0.0197 & 0.0249 & 0.6667 & 3 & GFD \\
8 & 0.4464 & 0.0308 & 0.4183 & 0.5833 & 4 & GFD \\
9 & 0.2548 & 0.0819 & 0.6590 & 1.0000 & 5 & ZER \\
10 & 0.0695 & 0.4496 & 0.2908 & 0.5000 & 4 & GFD \\
11 & 0.6254 & 0.0700 & 0.0586 & 0.8333 & 3 & GFD \\
12 & 0.2130 & 0.0409 & 0.4360 & 0.8333 & 5 & ZER \\
13 & 0.1986 & 0.0491 & 0.5046 & 0.2500 & 4 & GFD \\
14 & 0.4471 & 0.0000 & 0.7777 & 0.6667 & 5 & ZER \\
15 & 0.5845 & 0.0571 & 0.3412 & 0.1667 & 4 & GFD \\
16 & 1.0000 & 0.0246 & 0.0000 & 0.5000 & 6 & ZER \\
17 & 0.9060 & 0.2681 & 0.1051 & 0.1667 & 6 & ZER \\
18 & 0.8145 & 0.1727 & 0.0969 & 0.6667 & 3 & GFD \\
19 & 0.7535 & 0.1776 & 0.1795 & 0.2500 & 6 & ZER
\end{tabular}

From this classification we obtain a global hit ratio of 12.4688 , in percentage $65.63 \%$ versus the best result we obtained with previous methods, $59.57 \%$ of GFD. It is important to notice that the best result we can obtain, that is to choose for each image the best method that recognizes it, is about $75.90 \%$.

\section{Conclusion}

In this paper we introduced invariant features that may be used for shape based image retrieval. We considered Generic Fourier descriptors, Hu's moment invariants, Taubin's moment invariants Flusser's moment invariants, Zernike moments and Legendre moments. Classification based on morphological image features, combined with moment invariants described, showed effective image retrieval.

\section{References}

1. M. K. Hu. Visual pattern recognition by moment invariants. IRE Trans. Information Theory, 8:179-187, 1962.

2. S. A. Dudani, K. J. Breeding, and R. B. McGhe. Aircraft identification by moment invariants. IEEE Trans. Computers, 26:39-45, 1977.

3. S. O. Belkasim, M. Shridhar, and M. Ahmadi. Pattern recognition with moment invariants : a comparative study and new results. Pattern Recognition, 24:11171138, 1991.

4. R. Y. Wong and E. L. Hall. Scene matching with invariant moments. Computer Graphics and Image Processing, 8:16-24, 1978. 
5. A. Goshtasby. Template matching in rotated images. IEEE Trans. Pattern Analysis and Machine Intelligence, 7:338-344, 1985.

6. J. Flusser and T. Suk. A moment-based approach to registration of images with affinne geometric distortion. IEEE Trans. Geoscience and Remote Sensing, 32:382387, 1994.

7. R. Mukundan and K. R. Ramakrishnan. An iterative solution for object pose parameters using image moments. Pattern Recognition Letters, 17:1279-1284, 1996.

8. R. Mukundan and N. K. Malik. Attitude estimation using moment invariants. Pattern Recognition Letters, 14:199-205, 1993.

9. A. Sluzek. Identification and inspection of 2-d objects using new moment-based shape descriptors. Pattern Recognition Letters, 16:687-697, 1995.

10. F. El-Khaly and M. A. Sid-Ahmed. Machine recognition of optically captured machine printed arabic text. Pattern Recognition, 23:1207-1214, 1990.

11. K. Tsirikolias and vol. 26 pp. 877-882 1993. B. G. Mertzios, ". Statistical pattern recognition using efficient two-dimensional moments with applications to character recognition. Pattern Recognition, 26:877-882, 1993.

12. A. Khotanzad and Y. H. Hong. Invariant image recognition by zernike moments. IEEE Trans. Pattern Analysis and Machine Intelligence, 12:189-197, 1990.

13. J. Flusser and T. Suk. Affine moment invariants: A new tool for character recognition. Pattern Recognition Letters, , 15:433-436, 1994.

14. S. Maitra. Moment invariants. Proc. of the IEEE, 67:697-699, 1979.

15. T. M. Hupkens and J. de Clippeleir. Noise and intensity invariant moments. Pattern Recognition, 16:371-376, 1995.

16. L. Wang and G. Healey. Using zernike moments for the illumination and geometry invariant classiffication of multispectral texture. IEEE Trans. Image Processing, 7:196-203, 1998.

17. Y. Li. Reforming the theory of invariant moments for pattern recognition. Pattern Recognition, 25:723-730, 1992.

18. W. H. Wong, W. C. Siu, and K. M. Lam. Generation of moment invariants and their uses for character recognition. Pattern Recognition Letters, 16:115-123, 1995.

19. J. Flusser. On the independence of rotation moment invariants. Pattern Recognition, 33:1405-1410, 2000.

20. J. Flusser. On the inverse problem of rotation moment invariants. Pattern Recognition, 35:3015-3017, 2002.

21. M. R. Teague. Image analysis via the general theory of moments. J. Optical Soc. of America, 70:920-930, 1980.

22. A. Wallin and O. Kubler. Complete sets of complex zernike moment invariants and the role of the pseudoinvariants. IEEE Trans. Pattern Analysis and Machine Intelligence, 17:1106-1110, 1995.

23. M. K. Hu. Pattern recognition by moment invariants. Proc.IRE, 49, 1961.

24. J. F. T. Suk. Pattern recognition by affine moment invariants. Pattern Recognition, 26:167-174, 1993.

25. G. Taubin and D.B. Cooper. Geometric invariance in computer vision, chapter Object recognition based on moment (or algebraic) invariants, pages 375-397. MIT Press, 1992.

26. C-H. Teh and R.T. Chin. On image analysis by the method of moments. IEEE Trans. on Pattern Analysis and Machine Intelligence, 10(4):496-513, 1988.

27. M.R. Teague. Image analysis via the general theory of moments. Journal of the Optical Society of America, 70(8):920-930, 1979.

28. http://www.cs.rutgers.edu/pub/sven/rutgers-tools/.

29. http://www.lems.brown.edu/vision/software/. 